\title{
Non-ergodic behaviour in conformational transitions of single DNA molecules
}

\author{
Stefan Wennmalm, Lars Edman, Rudolf Rigler * \\ Department of Medical Biophysics, Karolinska Institute, S-171 77, Stockholm, Sweden
}

Received 16 December 1998

\begin{abstract}
Conformational fluctuations in single nucleic acid molecules have recently been observed through excited state lifetime measurements. Immobilisation of the sample molecule has also enabled direct observation of the fluorescence intensity fluctuations generated as the molecule switches between two conformations. As a probe for conformational fluctuations we use tetramethylrhodamine linked to a 217-bp DNA oligonucleotide. The measurements on this and similar systems reveal the existence of a distribution of reaction rates between the conformations. Here we report 37 detected single-molecule conformational fluctuations collected with the same immobilisation method as described earlier. Within the time window of observation the reaction rates differ between the molecules, but stay constant within a single molecule. The distribution of the relaxation rates between the molecules correspond to the distribution seen in a bulk measurement on a similar system. We therefore conclude that within the observation time window the single DNA molecules behave in a non-ergodic way. (c) 1999 Elsevier Science B.V. All rights reserved.
\end{abstract}

\section{Introduction}

The last years numerous reports showing the possibility of single-molecule detection (SMD) in solution and on surfaces, by near- and far-field microscopy have been published [1-6]. In addition, other SMD reports show that properties of molecules differ either between molecules $[7,8]$ or within a single molecule as a function of time [9-11]. Likely the explanation for changes in properties within a molecule is conformational. In the case of fluorescent molecules these changes shift the photophysical

\footnotetext{
* Corresponding author. E-mail: rudolf.rigler@mbb.ki.se
}

behaviour of the fluorophore. Recently several reports have shown single-molecule conformational fluctuations in a more controlled system [12-15]. A double stranded DNA with tetramethylrhodamine (TMR) attached through a carbon atom linker has been shown to fluctuate between two conformations; one where the TMR molecule is attached to a guanosine base and one where it is not. In a study of single tRNA $^{\text {Phe }}$ molecules where TMR was attached to 4-thiouridine a similar behaviour was seen [13]. Guanosine has a known quenching effect on rhodamine dyes and this effect is not obtained by other nucleotides [16-18]. The conformational fluctuations in these sample molecules have been detected either as the existence of separate excited state lifetimes in a single molecule $[12,13,15]$, or as direct shifts in the 
fluorescence intensity of the dye as it attaches or detaches to the specific quencher [14].

By the use of fluorescence correlation spectroscopy (FCS), bulk measurements in solution on a double stranded DNA molecule have revealed a distribution of reaction rates between the two conformations [12]. The question has been raised whether this is a distribution between molecules (heterogeneous case) or a distribution in time (homogeneous case). Different reaction rates can arise if one conformation of a molecule exists in several conformational substates $[19,20]$. For the question to be answered a direct observation of the individual transitions between the two conformations is necessary. Through immobilisation of a $217 \mathrm{bp}$ double stranded DNA on a glass surface via the biotin-streptavidin interaction this was accomplished, and within the time window of observation the obtained data supported the heterogeneous case [14]. It was however clear that either measurements over longer time ranges or more detections of the same kind were necessary for a final determination to be made. In a recent paper an expression was derived which describes the probability density for the fraction of time that one of two states is occupied during a given measuring interval. Application to data obtained on the present sample molecule supported the heterogeneous case [21]. Here we present data on the same sample molecule and with the same immobilisation method as was used in Ref. [14]. The relaxation rate of each detected molecule is calculated and the distribution is compared with the distribution analysis made on the similar system in Ref. [12].

\section{Materials and methods}

The sample molecule, a 217-bp double stranded DNA labeled with tetramethylrhodamine (TMR) through a six carbon atom linker at the 5-prime end, was obtained by PCR. As template in the PCR we used M13 mp18(+) strand DNA (7250 bases). As forward primer 5'-(TMR)-AAAGGGGGATCTGCTGCAAGGCG was used and as reversed primer 5'-(Bi)-GCTTCCGGCTCGTATGTTGTGTG was used. Streptavidin-coated coverslips were prepared, and through the biotin-streptavidin interaction the biotin end of the sample molecules was attached to the coverslips. PCR conditions, control analysis of the PCR product by use of FCS, preparation of streptavidin coated coverslips used for immobilisation, and immobilisation of DNA on coverslips are described in Ref. [14].

The experimental setup for FCS has been described in Ref. [1]. A Zeiss $40 \times$ NA 0.9 objective provided an excitation volume of $1 \mu \mathrm{m}$ diameter and $4 \mu \mathrm{m}$ length. A scanning table (ITK Lahnau, Germany; MC-2000), movable in three dimensions with $1 \mu \mathrm{m}$ steps, was used to move the coverslip relative to the excitation volume. In the search for single fluctuating DNA molecules a random spot on the coverslip surface was positioned into the excitation volume after which the intensity measurement was immediately started. If a single molecule was found the search procedure could be interrupted and the intensity measurement continued on the same spot for as long as necessary. The fluorescence intensity measurements were taken with a digital correlator (ALV, Germany; ALV-5000). An excitation intensity such that the average fluorescence intensity did not exceed $3 \mathrm{kHz}$ per molecule was used.

\section{Results}

The evidence for a single-molecule detection (SMD) is a constant fluorescence intensity which suddenly and irreversibly drops to background level as photo-induced decomposition occurs [22]. After an assumed decomposition intensity collection was continued for an additional $30 \mathrm{~s}$ in order to confirm that the molecule was really decomposed. More than 100 detections were made where the survival time (time before decomposition occurs) was too short for any transitions to be observed. In the case that the molecule is fluctuating between two conformations, fluorescence intensity fluctuations between a low and a high level can be observed before decomposition occurs (Fig. 1a-d). In 37 cases fluorescence fluctuations were detected.

In most cases the fluorescence intensity difference between the low fluorescence level and the background intensity could not be distinguished; however, in a few cases the difference was clear (Fig. 1a and c). The occupation time (alternatively on- or 

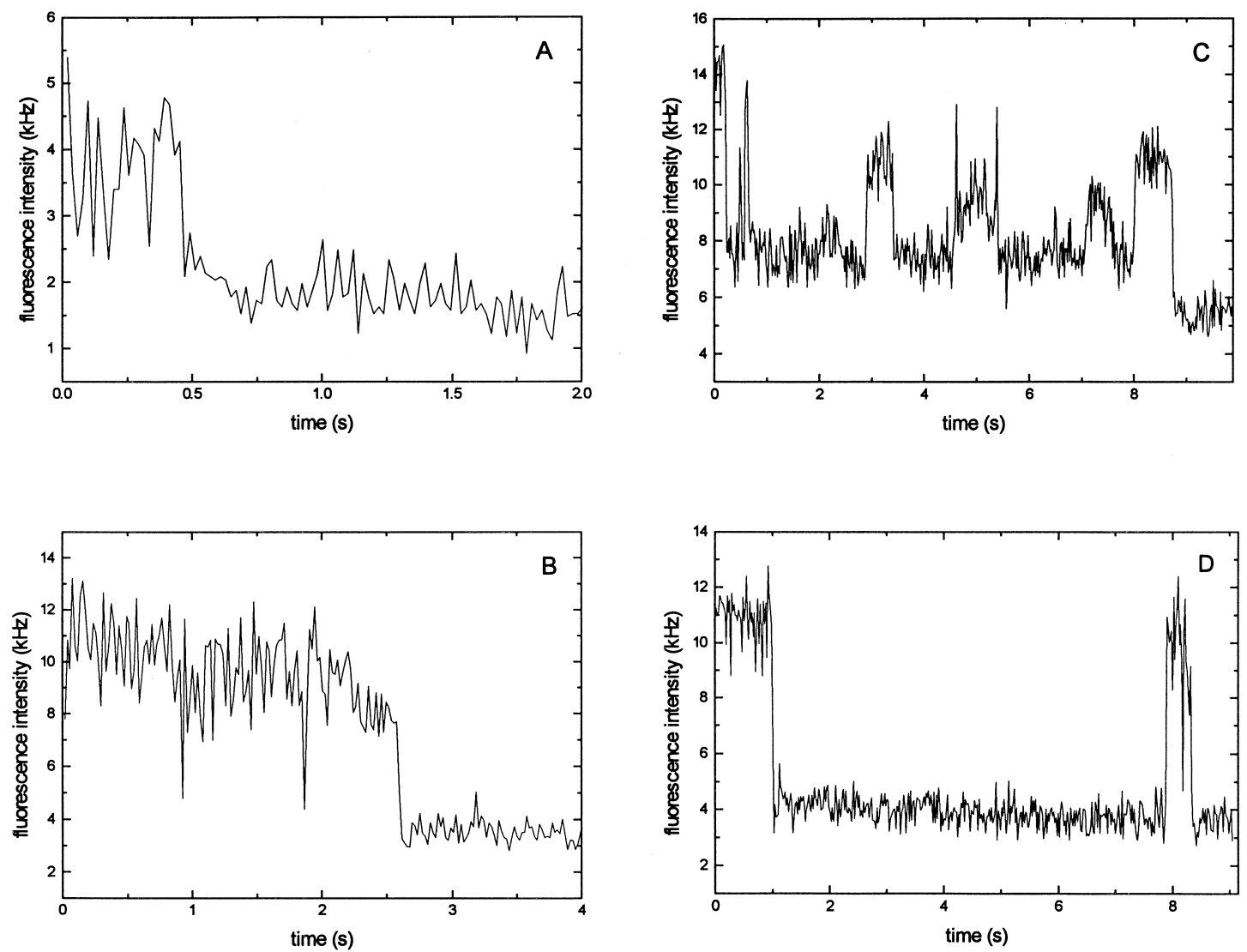

Fig. 1. (a) The TMR labeled DNA molecule undergoes eight transitions before decomposition occurs after $0.5 \mathrm{~s}$. (b) An example of a molecule with a fast on-rate and a slow off-rate. (c) The height of the fluorescence levels indicates that at least two quenched states exist in addition to the non-quenched state. (d) Two consecutive occupation times of 7 and $0.02 \mathrm{~s}$, indicating a mixture of reaction rates within the same molecule. The relaxation rates in (a), (b) (c) and (d) are 54, 39, 10 and $27 \mathrm{~s}^{-1}$, respectively.

off-time, depending on whether the molecule is in the high or low fluorescent conformation) is defined as the time spent in one conformation between two consecutive transitions. Occupation times shorter than the $20 \mathrm{~ms}$ resolution of the intensity trace will not be seen, or appear only as peaks (or dips) with a smaller amplitude. Such fluctuations were therefore not taken into account, and accordingly the shortest occupation time recorded was $20 \mathrm{~ms}$ and the fastest reaction rate was $50 \mathrm{~s}^{-1}$. Histograms of the on- and off-times of all detected transitions were calculated (Fig. 2a and b) and analyzed according to

$$
d_{1}(t)=a_{1} \exp \left(-t / \tau_{\mathrm{s}}\right) \exp \left(-t / \tau_{\mathrm{occ}}\right),
$$

where $\tau_{\text {occ }}$ is the characteristic occupation time and $\tau_{\mathrm{s}}$ is the effective survival time of the molecules.
The survival time of the molecules has to be considered in the analysis since it affects the probability of detecting transitions. The survival time has been analyzed [22] and was fixed to $2.7 \mathrm{~s}$. Both histograms required an additional term for acceptable fits to be achieved. Thus Eq. (1) was extended to

$$
\begin{aligned}
d_{2}(t)= & a_{1} \exp \left(-t / \tau_{\mathrm{s}}\right)\left[a_{2} \exp \left(-t / \tau_{\text {occ. } 1}\right)\right. \\
& \left.+\left(1-a_{2}\right) \exp \left(-t / \tau_{\text {occ } .2}\right)\right] .
\end{aligned}
$$

However, due to the limited amount of data points the additional occupation time could only be estimated. The average occupation times were

$$
\begin{aligned}
& \tau_{\text {on }, 1}=0.16 \pm 0.07 \mathrm{~s} \quad \text { and } \quad \tau_{\text {on, }, 2}=4 \pm 4 \mathrm{~s} \\
& \tau_{\text {off }, 1}=0.03 \pm 0.009 \mathrm{~s} \quad \text { and } \quad \tau_{\text {off }, 2}=4 \pm 4 \mathrm{~s} .
\end{aligned}
$$


The normalized amplitudes for the shorter occupation times were 0.86 for the on-times and 0.94 for the off-times. Analysis of histograms $2 \mathrm{a}$ and $2 \mathrm{~b}$ according to a stretched exponential model was attempted but did not give acceptable fits.

The reaction on-rate of a molecule is defined as the average of the inverse of the occupation time in the low fluorescent conformation, and analogous for the reaction off-rate. The relaxation rate of a molecule is defined as the sum of the reaction rates, $k_{\mathrm{r}}=k_{\text {on }}$ $+k_{\text {off }}$. In a bulk measurement where the fluctuations are investigated through calculation of the autocorrelation of the fluorescence fluctuations from many molecules, only the relaxation rates and not the
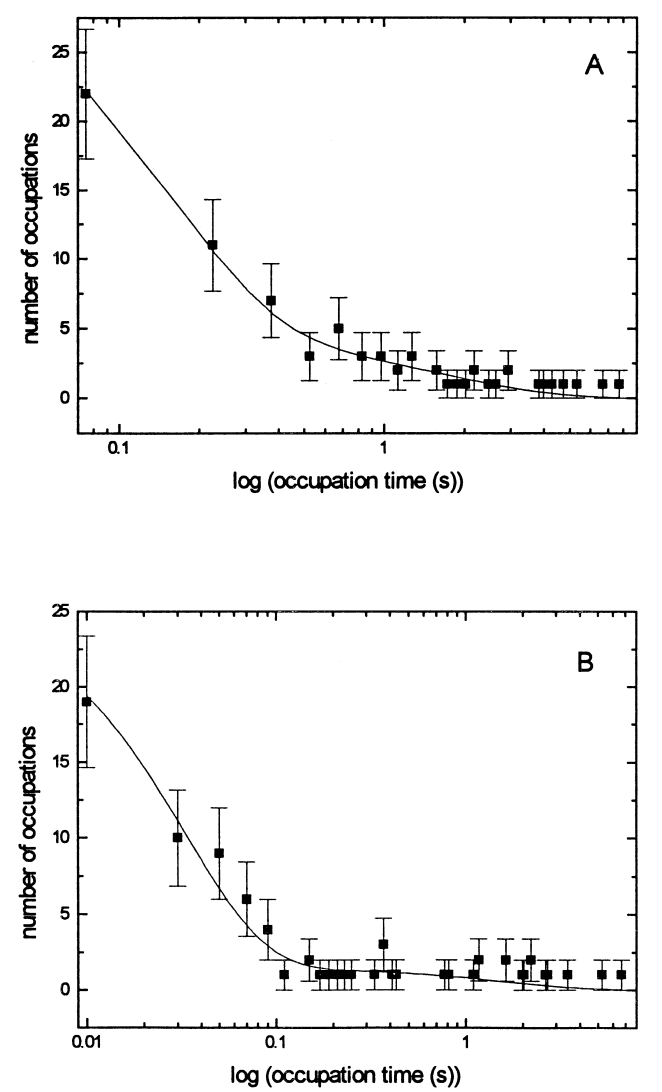

Fig. 2. The effective survival time $\tau_{\mathrm{s}}$ has been analyzed [22] and was fixed to $2.7 \mathrm{~s}$. (a) shows a histogram of all on-times and (b) shows a histogram of all off-times. In (a) the dominating faster on-time of $0.16 \mathrm{~s}$ has a normalized amplitude of 0.86 and in (b) the dominating off-time of $0.03 \mathrm{~s}$ has an amplitude of 0.94 .

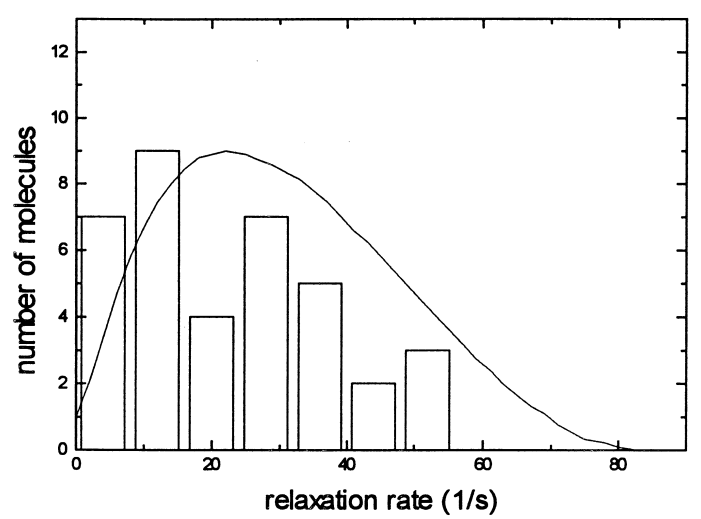

Fig. 3. A histogram of the relaxation rates of the 37 molecules corresponding to the distribution of relaxation rates found in a bulk measurement on a similar sample molecule [12]. The mean relaxation rate of the 37 molecules is $22.3 \mathrm{~s}^{-1}$ which is close to the peak in the bulk measurement distribution.

individual reaction rates can be obtained [12]. For comparison, the relaxation rate for each molecule was therefore obtained and a histogram was calculated (Fig. 3). The inserted curve is the distribution $p_{i}$ (Eq. (5)) of the relaxation rates found in a solution bulk measurement on a similar sample molecule [12].

The relaxation rate for the similar sample molecule in bulk was obtained by FCS. In order to achieve an acceptable fit of the data from diffusing molecules undergoing conformational fluctuations, a relaxation factor consisting of a distribution of exponentials was required (formula (5))

$$
\sum_{i=1}^{N} p_{i}\left[1+A \exp \left(-k_{i} T\right)\right] \text {. }
$$

In formula (5) $N$ is the average number of molecules in the excitation volume, $k$ is the relaxation parameter and $A=K[(1-Q) /(1+Q K)]^{2}$, where $K$ is the equilibrium constant between the two conformations and $Q$ is the quantum yield ratio.

\section{Discussion}

The histogram of the individual relaxation rates for all molecules show a distribution which ranges from 1 to $50 \mathrm{~s}^{-1}$. In addition, there is a clear 
correspondence between the relaxation rate histogram and the distribution of rates obtained from bulk measurement (Fig. 3). For the sample molecule, this is a strong indication in favor of the heterogeneous explanation for the distribution of relaxation rates. Actually the heterogeneity of the system can be observed directly in the intensity traces. For example, there is a clear difference in the rates between the fast fluctuations in Fig. 1a and the slower ones in Fig. 1c; however, within the two molecules the rates are constant. A debated question has been whether the behaviour of molecules can be expected to be the same in solution and on surfaces. Despite the fact that in the many-molecule experiment a slightly different primer was used, which in addition was not attached to a surface but diffusing freely in solution [12], the relaxation rates of the two systems correspond well. Thus at least for this sample molecule the dynamics observed in solution seem to be unaffected by attachment on a surface.

The $20 \mathrm{~ms}$ resolution in the intensity traces sets an upper limit of $100 \mathrm{~s}^{-1}$ for the relaxation rates. However, for a single molecule to display such a high relaxation rate requires a combination of reaction rates and survival time which has very low probability. In practice relaxation rates $>50 \mathrm{~s}^{-1}$ will not be observed, and thus a comparison of bulk and single-molecule data cannot be made for relaxation rates exceeding $50 \mathrm{~s}^{-1}$ (Fig. 3). In a recent paper a model describing the probability density that one of two states is occupied during a defined measuring interval was derived [21]. In the analysis where the model was applied to fluctuating molecules of the same type as used here, a time resolution of $0.25 \mathrm{~s}$ was used. Even though we use a much higher resolution of $20 \mathrm{~ms}$, the data presented here and the probability density model analysis used in Ref. [21] both indicate a heterogeneous distribution of reaction rates.

From the intensity traces some other information is obtainable in addition to the finding that the heterogeneous distribution of the relaxation rates correspond to the distribution seen in bulk measurements. For example, a single molecule can have one constant on-rate and a different constant off-rate (Fig. 1b), a phenomenon with the principal explanation that the free energy of the quenched conformation is closer to the top of the energy barrier between the conformations than the free energy of unquenched TMR. The relaxation rate of the trace in Fig. $1 \mathrm{~b}$ is $39 \mathrm{~s}^{-1}$, which gives no indication of the existing slow off-rate of $1 \mathrm{~s}^{-1}$. This is a typical example of the limitations of bulk measurements. Even though a distribution in the relaxation rates can be revealed and the equilibrium constant between the conformations can be estimated, the question of how the distributed on- and off-rates are put together in a single molecule will remain unknown. In principle, a distribution in the relaxation rates can origin from the existence of one constant on-rate - common for all molecules - and a distribution in the off rates, or vice versa. In the data molecules with on-rates which were more than three times as large as the off-rates were more common than molecules where the two rates were of equal magnitude. Very few molecules were seen which had a fast off-rate and a slow on-rate. This is in accordance with the mean fraction of time of 0.22 spent in the quenched conformation obtained from excited state lifetime bulk measurements.

The histograms of the on- and off-occupation times showed a decay according to a two exponential term model (Fig. 2a and b). If only the two main occupation times $\tau_{\text {on }}=0.156 \mathrm{~s}$ and $\tau_{\text {off }}=0.033 \mathrm{~s}$ are considered, the fraction of time spent in the low fluorescent conformation is 0.17 . This corresponds well with the relative fraction of 0.22 of the quenched conformation found in an excited state lifetime bulk measurement on the sample molecule [14]. The necessity of two exponential terms in both histograms indicates that all detected occupations do not origin from the same single exponential process. Base-pair opening rates in the time range $10^{-3} \mathrm{~s}$ have been observed [23] and is a likely explanation for the two main occupation times. However, considerably longer times such as the smaller components of $\sim 4 \mathrm{~s}$ (Fig. 2) have to our knowledge never been observed. Although we do not know the details of such slow fluctuations, they could be explained by simultaneous breaking of hydrogen bonds, e.g. base-pair opening at the 5'- and 3 '-end of the DNA molecule [24].

The ergodic hypothesis assumes that measurement of the time average of a quantity on a single member of an ensemble will give the same result as a short measurement of the average of the same quantity on the whole ensemble. However it is for example well 
known that proteins can fold into deep free energy minima in numerous different ways, and this can affect the availability of an active site in the protein to surrounding molecules. If the proteins do not change their structures over time scales which by far exceeds the characteristic reaction time, they will in practice constitute a heterogeneous ensemble. Whether the system should be regarded as nonergodic will then be a question of how long the dynamics of a single protein can be observed. If the energy landscape of our sample molecule is such that the dye can be quenched by the guanosine from more than one free energy minimum (conformational substate), a mixture of reaction rates in a single molecule can be seen. If several such substates exist all molecules can in principle behave identically and still generate a distribution of relaxation rates, since each molecule behaves as the ensemble (ergodicity) $[19,20]$. Depending on the shape of the energy barriers the dye could either do many on- and off-transitions into one substate before changing to another substate, or the dye could use the different substates more randomly. The latter cannot be the case for our sample molecules since all except one trace do not show a mixture of occupation times. The former possibility however cannot be excluded. In one trace two consecutive occupation times differed by more than a factor of 300 (Fig. 1d). A simulation of single exponentially distributed occupation times gives that the probability of two such consecutive occupation times is 0.007. Even if the 18 detections that had equal prerequisites (sufficient survival times) are taken into account, the probability is still 0.9 that such an event will not be seen in single exponentially distributed occupation times. It is therefore possible that the trace in Fig. 1d shows a shift from a slower to a faster mode of fluctuation. If that is the case the system may still be regarded as ergodic if it is only given enough time to go through all different reaction rate modes. In a recent work [25] fluorescence fluctuations due to enzymatic turnovers of single cholesterol oxidase molecules were analyzed. A distribution in the turnover number of the enzyme due to conformational fluctuations was observed. A two conformational state model was sufficient for explaining the distributed turnover number; however, even a broad a distribution of substates could not be excluded.
In the conformation where TMR is attached to the guanosine base an electron transfer mechanism will compete with the photon emission process. Thus the attachment to guanosine results for TMR in an additional excited state lifetime of $1.36 \mathrm{~ns}$ as compared to $4.06 \mathrm{~ns}$ for free TMR [14]. As a consequence the fluorescence intensity of TMR when attached to guanosine is reduced by a factor of three. The effect is for TMR and other rhodamine dyes only obtained by guanine [16-18]. In a stack between Rh6G and guanine in aqueous solution a reduction of the lifetime of Rh6G from $\sim 4$ ns to 200 ps has been observed [16]. Such a strong quenching has not been observed in excited state lifetime measurements on the present sample molecule. However, in most traces the fluorescence intensity difference between the quenched conformation and the background intensity could not be distinguished, which could be the result of a heavily quenched low fluorescent conformation. Likely the explanation for different quenching strengths is the distance dependence of the electron transfer probability, and the different ways in which the dye can attach to the quencher. In three traces two separate fluorescence levels were seen in addition to the low fluorescent conformation (Fig. 1c). If TMR can be quenched from several conformational substates it is likely that the quenching strength is not equal at the different substates. The existence of more than one quenched conformation in a similar sample molecule has recently been proposed by Eggeling et al. [15].

A prerequisite for observing single-molecule fluctuations is a sufficient survival time of the fluorescent probe. We used an excitation intensity such that the average fluorescence intensity of the molecules was $3.4 \mathrm{kHz}$ and the characteristic survival time 4.1 s [22]. Likely the more than 100 detected molecules that showed no fluctuations did so because their survival times were too short in comparison to their occupation times. However, we cannot exclude that some molecules were truly non-fluctuating. Eqs. (1) and (2) describe the joint probability that a detected molecule survives a certain time and displays a certain occupation time. A $50 \%$ probability of detecting two consecutive transitions (needed for a fluctuation to be recorded) requires that the survival time $\tau_{\mathrm{s}}$ is 1.5 times the occupation time $\tau_{\text {occ }}$. Thus the effective survival time in Eqs. (1) and (2) is fixed to 
$2.7 \mathrm{~s}$, as compared to the characteristic survival time of the detected molecules of $4.1 \mathrm{~s}$.

In conclusion, with the time window of observation and the limiting survival time of the fluorophores, we report a non-ergodic behaviour in the conformational fluctuations of single DNA molecules. The average relaxation rates of $37 \mathrm{de}-$ tected molecules were distributed with a mean of 22 $\mathrm{s}^{-1}$, and the distribution found correspond to the distribution seen in a bulk measurement on a similar sample molecule. The average fraction of time spent in the quenched conformation was 0.17 which corresponds well to the fraction of 0.22 observed in excited state lifetime bulk measurements on the sample molecule. In addition, to the main occupation times a small component of $\sim 4 \mathrm{~s}$ was observed. Such times deviate from base-pair opening rates previously observed [23], and have to be explored further. Examples of single molecules which exist in conformations with different quenching intensities have been observed. In addition, a possible indication of a mixture of reaction rates in a single molecule has been seen. Such a behaviour can arise if a molecule inherits several conformational substates within a single conformation. It seems however that the sample molecule will not go randomly into either one of the quenched substates, but instead fluctuate in and out of one substate for a while before changing to another, as is suggested by the heterogeneous distribution of the molecules. This is in line with theoretical conclusions of a very rough conformational landscape for complex molecules such as proteins [26].

\section{Acknowledgements}

We thank Dr. I. Zelikman (Pharmacia Diagnostics $\mathrm{AB})$ for help with streptavidin coating, Fredrik Thamsen for help with data collection and Dr. Per Thyberg for discussions. This study was supported by grants from the Swedish Natural Science Research Council and Swedish Research Council for Engineering Sciences.

\section{References}

[1] R. Rigler, J. Widengren, Ü. Mets, in: O.S. Wolfbeis (Ed.), Fluorescence Spectroscopy: New Methods and Applications, Springer, Berlin, 1992, pp. 13-24.

[2] W.P. Ambrose, P.M. Goodwin, J.C. Martin, R.A. Keller, Science 265 (1994) 364-367.

[3] Ü. Mets, R. Rigler, J. Fluoresc. 4 (1994) 259-264.

[4] J.J. Macklin, T.D. Trautman, L.E. Brus, Science 272 (1996) $255-258$.

[5] Th. Schmidt, G.J. Schutz, W. Baumgartner, H.J. Gruber, H. Schindler, Proc. Natl. Acad. Sci. USA 93 (1996) 2926-2929.

[6] X. Xu, E.S. Yeung, Science 275 (1997) 1106-1109.

[7] J.K. Trautman, J.J. Macklin, L.E. Brus, E. Betzig, Nature (London) 369 (1994) 40-42.

[8] Q. Xue, E.S. Yeung, Nature (London) 373 (1995) 681-683.

[9] X.S. Xie, R.C. Dunn, Science 265 (1994) 361-364.

[10] S.X. Xie, Acc. Chem. Res. 29 (1996) 598-606.

[11] R.M. Dickson, A.B. Cubitt, R.Y. Tsien, W.E. Moerner, Nature (London) 388 (1997) 355-358.

[12] L. Edman, Ü. Mets, R. Rigler, Proc. Natl. Acad. Sci. USA 93 (1996) 6710-6715.

[13] Y. Jia, A. Sytnik, L. Li, S. Vladimirov, B.S. Cooperman, R.M. Hochstrasser, Proc. Natl. Acad. Sci. USA 94 (1997) 7932-7936.

[14] S. Wennmalm, L. Edman, R. Rigler, Proc. Natl. Acad. Sci. USA 94 (1997) 10641-10646.

[15] C. Eggeling, J.R. Fries, L. Brand, R. Günther, C.A.M Seidel, Proc. Natl. Acad. Sci. USA 95 (1998) 1556-1561.

[16] J. Widengren, J. Dapprich, R. Rigler, Chem. Phys. 216 (1996) 417-426

[17] C.A.M. Seidel, A. Schulz, M.H.M. Sauer, J. Phys. Chem. 100 (1996) 5541-5553

[18] M. Sauer, K.-T. Han, R. Muller, S. Nord, A. Schulz, S. Seeger, J. Wolfrum, J. Arden-Jacobi, G. Deltau, N.J. Marx, C. Zander, K.H. Drexhage, J. Fluoresc. 5 (3) (1995) 247-261.

[19] H. Frauenfelder, in: K.L. Ngai (Ed.), Glasses and Glass Forms, Mater. Res. Soc., Pittsburgh, PA, 1997.

[20] H. Frauenfelder, S.G. Sligar, P.G. Wolynes, Science 254 (1991) 1598-1603.

[21] L. Edman, S. Wennmalm, F. Thamsen, R. Rigler, Chem. Phys. Lett. 292 (1998) 15-21.

[22] S. Wennmalm, R. Rigler, J. Phys. Chem. (1999, in press).

[23] T.M. Nordlund, P. Wu, S. Andersson, L. Nilsson, R. Rigler, A. Gräslund, L.W. McLaughlin, B. Gildea, Proc. SPIE (Soc. Photo-Opt. Instrum. Eng.) (1990) 344-353.

[24] W. Saenger, Principles of Nucleic Acid Structure, Springer, Berlin, 1984.

[25] H.P. Lu, L. Xun, X.S. Xie, Science 282 (1998) 1877-1882.

[26] J. Bryngelson, P.G. Wolynes, J. Phys. Chem. 93 (1989) 6902. 